\title{
Maximum Life-Time Localized Broadcast Routing in MANET
}

\author{
Ruiqin Zhao, Aijun Wen, Zengji Liu, and Peng Yue \\ State Key Lab. of Integrated Service Networks, \\ Xidian University, Xi' an 710071 China \\ rqinzhao@gmail.com, \{ajwen, zjliu,pyue\}@xidian.edu.cn
}

\begin{abstract}
Added delay strategy can be used to solve the broadcast storm problem of ordinary broadcast mechanism (OBM) and maximize network life-time. Available added delay strategies take into account the distance and/or the residual energy. In this paper, we propose a new added delay strategy-Maximum Life-time Localized Broadcast $\left(\mathrm{ML}^{2} \mathrm{~B}\right)$. As the node's number of neighbors that have not received the broadcast message (we call it coverage degree) can better describe the coverage rate, $\mathrm{ML}^{2} \mathrm{~B}$ takes the coverage degree rather than the distance into account. $\mathrm{ML}^{2} \mathrm{~B}$ also takes the residual energy into account as other strategies do. $\mathrm{ML}^{2} \mathrm{~B}$ only need one-hop neighbor information to find the coverage degree, so $\mathrm{ML}^{2} \mathrm{~B}$ is a distributed protocol and the overhead is small. Simulation results show that, as compared with OBM, $\mathrm{ML}^{2} \mathrm{~B}$ can save at least $50 \%$ rebroadcast, its maximum end-to-end delay is lower, its reachability is the same, and its network life-time is two times longer.
\end{abstract}

Keywords: Broadcast, life-time, localized, $\mathrm{ML}^{2} \mathrm{~B}$, wireless ad hoc networks.

\section{Introduction}

The issue of energy-efficient communication in ad hoc networks has been attracting attention of many researches during last several years. Broadcasting is a common operation in ad hoc networks which is used not only for distributing the data among all network devices, but also for spreading the control information, e.g., for maintaining routes between devices and updating their states.

The straightforward way of broadcast in MANET is flooding which also is known as ordinary broadcast mechanism (OBM). OBM will cause serious broadcast redundancy, which is referred to as broadcast storm problem [1]. A satisfying broadcast strategy should be able to reduce the broadcast redundancy effectively, not only for the saving of bandwidth, but also for the saving of energy, as both bandwidth and energy are valuable resources in MANET.

With the aim of solving the broadcast storm problem and maximizing the network life-time, we propose an efficient broadcast algorithm-Maximum Life-time Localized Broadcast $\left(\mathrm{ML}^{2} \mathrm{~B}\right)$ for MANET, which possesses the following properties:

1. Localized algorithm. Each node makes the decision of rebroadcast according to its one-hop local information. $\mathrm{ML}^{2} \mathrm{~B}$ need not maintain any global topology information at each node, so the overhead is small. 
2. Effective Coverage adaptive broadcast strategy. To reduce the redundancy of rebroadcast, nodes with larger coverage degree are selected as forward nodes with higher priority. The coverage degree is the number of left neighbors that have not received the broadcast message, it describes the effective coverage rate of the broadcast node more accurately than the distance of the node, and reduces broadcast redundancy more effectively.

3. Energy-efficient approach. Small algorithm overhead and reduced broadcast redundancy result in the reduction of energy consumption. $\mathrm{ML}^{2} \mathrm{~B}$ also takes residual energy of the node into consideration when selecting rebroadcasting node to maximize network life-time,

\section{Related Works}

Various approaches have been proposed to solve the broadcast storm problem of ordinary broadcast mechanism, generally they can be classified into two categories: energy-saving methods and non energy-saving methods.

Non energy-saving methods are designed with the aim of alleviating the broadcast storm problem by reducing redundant broadcasts. As in [2]-[6], each node computes a local cover set consisting of as less neighbors as possible to cover its whole 2-hop coverage area by exchanging connectivity information with neighbors. Several methods [3], [5]-[8] require each node know its k-hop ( $\mathrm{k}>=2$ ) neighbor information. These non energy-saving methods need heavy overhead to maintain the fresh k-hop ( $\mathrm{k}$ $>=2$ ) neighbor information and they consume much energy at each node. Some methods proposed in [1] select forward node based on probability, which cannot guarantee the reachability of the broadcast.

Most proposed energy-saving broadcast methods are centralized, which require the topology information of the whole network. They try to find a broadcast tree such that the energy cost of the broadcast tree is minimized. Methods in [9]-[12] are based on geometry information of the network, and others [13]-[16] are based on graph theory to compute the minimum energy tree. Since the centralized method will cause much overhead in MANET, some localized versions of the above algorithms have been proposed recently. [17] reduces energy consumption by taking advantage of the physical layer design. [18] finds the network topology in a distributed way, it requires every node to maintain the network topology, and the overhead is obviously more than a localized algorithm. The method proposed in [19] requires that each node must be aware of the geometry information within its 2-hop neighborhood. It results in more control overhead and energy cost than the thorough distributed algorithm that requires only one-hop neighbors' information.

\section{System Model}

The MANET can be abstracted as a graph $G(V, E)$, in which $V$ is the set of all the nodes in the network and $E$ consists of edges in the graph. We assume all links in the 
graph are bidirectional, and the graph is in a connected state. Given a node $i$, time $t$ is 0 when it receives the broadcasted message for the first time. The residual energy of node $i$ is $e(i, t) . r$ is the radius of the coverage of the node. $n b(i)$ is the one-hop neighbor of node $i$, and $N B(i)$ is the set of all neighbors of node $i$. We assume each node knows its own position information by means of GPS or other instruments. It can obtain its one-hop neighbors' information like most location-aided routing [20] of MANET do. Residual energy is also known at each node locally.

For $\forall i \in V$, several variables are defined as follows:

- Uncovered set $U C(i, t)$, consists of one-hop neighbors that have not been covered by a certain forward node of the broadcasted message or the broadcast originator, before $t$.

- Coverage Degree $d(i, t)$, is the number of nodes belonging to $U C(i, t)$ at $t$. $d(i, t)$ implies the rebroadcast efficiency of node $i$. If $d(i, t)$ is below a threshold before its attempt to rebroadcast the broadcasted message, node $i$ would abandon the rebroadcast.

- Up-link forward node $u f(i, t)$, is the $n b(i)$ that rebroadcasts or broadcasts the message which is received by node $i$ at $t(0 \leq t \leq D(i))$. During the period of $0<t \leq D(i)$, it may receive several copies of the same broadcasted message from different up-link forward nodes ( $D(i)$ is the added delay of node $i$ ).

- Up-link forward set $U F(i, t)$, is the set of all up-link forward nodes of node $i$ before $t$. If it has received the same broadcasted message for $k$ times before $t(t \leq D(i))$, its up-link forward set can be expressed as:

$$
U F(i, t)=\left\{u f\left(i, t_{0}\right), u f\left(i, t_{1}\right), u f\left(i, t_{2}\right) \ldots u f\left(i, t_{k-1}\right)\right\},(k \geq 1) .
$$

(where $t_{0}, t_{1}, t_{2} \ldots$, and $t_{k-1}\left(t_{k-1} \leq t\right)$ records the time node $i$ receives the $1 \mathrm{st}, 2 \mathrm{nd}, 3 \mathrm{rd} \ldots$, and $k$ th copy of the same broadcasted message).

\section{Maximum Life-Time Localized Broadcast $\left(\mathrm{ML}^{2} \mathrm{~B}\right)$ Algorithm}

\subsection{The Length of Added Delay $D(i)$}

Utilization of added delay in broadcast is to reduce the redundancy of nodes' rebroadcast and energy consumption. When node $i$ receives a broadcasted message for the first time, it will not rebroadcast it as OBM. It defers a period of $D(i)$ before its attempt to do the rebroadcast. Even when $D(i)$ expires, the node will not rebroadcast it urgently until the node coverage degree $d(i, D(i))$ is larger than the abandoning threshold $n$. During the interval of $0 \leq t \leq D(i), \forall$ node $i$ could abandon its attempt to rebroadcast the message as soon as its node coverage degree $d(i, t)$ is equal to 
or below the threshold, thus reducing the rebroadcast redundancy and energy consumption largely.

Nodes with larger added delay have a higher probability of receiving multiple copies of a certain broadcasted message from different up-link forward nodes, before they attempt to rebroadcast. Each reception of the same message decreases the node coverage degree, thus making nodes with large added delay rebroadcast the message with little probability. However nodes with little added delay may rebroadcast the message quickly. We assign little added delay or no-delay to nodes with high rebroadcast efficiency and enough residual energy, large added delay to nodes with large rebroadcast redundancy. To formulate the rebroadcast efficiency, two metrics are presented as follows:

$$
f_{d}(i)=\frac{a-d(i, 0)}{a}, \quad\left(0 \leq f_{d}(i) \leq 1\right) .
$$

Formula (2) is the node coverage degree metric. $a$ is the maximum node coverage degree. It can be induced from the formula that less $f_{d}(i)$ results in a higher rebroadcast efficiency.

To maximize the network life-time, we present the second metric-energy metric. If the residual energy at a node is smaller than an energy threshold $E_{T}$, it refuses to forward the broadcasted message. Otherwise, the node calculates the added delay based on formula (3), where $E^{\prime}$ is the maximum energy. $E_{T}$ is used to prevent nodes with little energy from dying.

$$
f_{e}(i)=\frac{E^{\prime}-e(i, 0)}{E^{\prime}-E_{T}}, \quad\left(E_{T} \leq e(i, 0) \leq E^{\prime}\right) .
$$

$\mathrm{ML}^{2} \mathrm{~B}$ first introduces a new metric $f(d(i, 0), e(i, 0))$ for the selection of rebroadcast node in MANET. It incorporates the two metrics presented above together to select rebroadcast nodes with goals of obtaining low rebroadcast redundancy, high reachability, limited latency, and maximized network life-time. We propose two different ways to combine node coverage degree and residual energy metrics into a single synthetic metric, based on the product and sum of the three metrics respectively. The synthetic product metric of delaying are given by formula (4). The sum metric is shown by formula (5) by suitably selected values of the two factors: $\alpha$ and $\beta$.

$$
\begin{gathered}
f^{\text {pro }}(d(i, 0), e(i, 0))=f_{d}(i) f_{e}(i) . \\
f^{\text {sum }}(d(i, 0), e(i, 0))=\alpha f_{d}(i)+\beta f_{e}(i) .
\end{gathered}
$$

We compute the added delay with the following formula:

$$
D(i)=D \cdot f(d(i, 0), e(i, 0)) \text {. }
$$


$D$ defines the maximum added delay. $f(d(i, 0), e(i, 0))$ is the synthetic metric shown by formula (4) or (5). Hence, based on formulas (2)-(6), we can get product and sum versions of the added delay are:

$$
\begin{gathered}
D^{\text {pro }}(i)=\frac{D[a-d(i, 0)]\left[E^{\prime}-e(i, 0)\right]}{\left(E^{\prime}-E_{T}\right) a} . \\
D^{\text {sum }}(i)=D\left(\frac{\alpha[a-d(i, 0)]}{a}+\frac{\beta\left[E^{\prime}-e(i, 0)\right]}{E^{\prime}-E_{T}}\right) .
\end{gathered}
$$

\subsection{Algorithm Description}

$\mathrm{ML}^{2} \mathrm{~B}$ is a thorough distributed broadcast routing protocol, in which each node requires only its one-hop local information to fulfill a broadcast task. The thorough distributed and localized nature of $\mathrm{ML}^{2} \mathrm{~B}$ could relieve the heavy overhead presented in most proposed energy-saving methods. The goal of a broadcast routing algorithm is to carry broadcasted messages to each node in network with as less rebroadcast redundancy as possible, satisfied reachability and maximized life-time of network. $\mathrm{ML}^{2} \mathrm{~B}$ is designed with the goal in mind. Let $s$ be the broadcast originator, the algorithm flow for $\forall i \in(V-\{s\})$ may be formalized as follows:

Step 0: Initialization: $j=-1, D(i)=D, U F(i, 0)=\varnothing$.

Step 1: If node $i$ receives the broadcasted message $M_{s}$, go to step 2; else if $j \geq 0$, go to step 7, else the node is idle, and stay in step 1 .

Step 2: Check the node ID of the originator $s$ and the message ID. If $M_{s}$ is a new message, go to step 3; else, node $i$ has received repeated $M_{s}$, then let $j=j+1$, and go to step 4 .

Step 3: Let $t=0$, and the system time begins. Let $j=0$, where $j$ indicates the times of the repeated reception of $M_{s}$. Let $U C(i, 0)=N B(i)$. Thus, $d(i, 0)$ equals the number of all neighbors. If $e(i, 0)$ is smaller than an energy threshold $E_{T}$, node $i$ abandons its attempt to rebroadcast, and go to step 9 .

Step 4: Let $t_{j}=t$, and use $p_{t_{j}}$ to mark the previous-hop node of $M_{s}$. $p_{t_{j}}$ transmits $M_{s}$ at $t_{j}$. We assume the propagation delay can be omitted. Then we get $u f\left(i, t_{j}\right)=p_{t_{j}}$, where $p_{t_{j}}$ is the $j$ th up-link forward node of node $i$. Add $p_{t_{j}}$ to up-link forward set $U F(i, t)$ at last.

Step 5: Based on the locally obtained position of $u f\left(i, t_{j}\right)$, node $i$ computes the geographical coverage range of $u f\left(i, t_{j}\right)$ which is expressed as $C\left(i, t_{j}\right)$. Then it updates $U C\left(i, t_{j}\right)$ by deleting nodes that locate in $C\left(i, t_{j}\right)$ from $U C\left(i, t_{j}\right)$, which is shown in Fig.1. Based on the updated $U C\left(i, t_{j}\right)$, node $i$ could calculate its coverage 


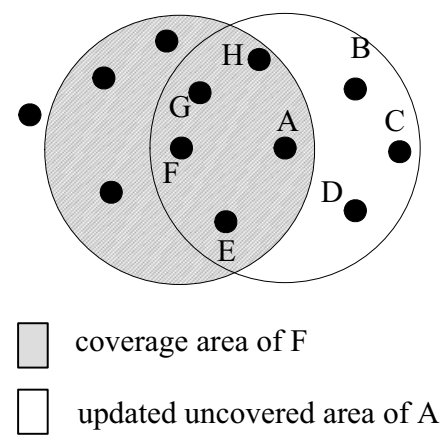

Fig. 1. Node $\mathrm{F}$ rebroadcasts $M_{s}$ at time $t$. When receives the message, node A updates its uncovered set by deleting node E, F, G and $\mathrm{H}$ which are covered by node F.

degree $d\left(i, t_{j}\right)$. If $d\left(i, t_{j}\right) \leq n$, it abandons its attempt to rebroadcast, and go to step 9; else if $j>0$, go to step 7 .

Step 6: $j=0$ means node $i$ has received $M_{s}$ for the first time. It calculates its added delay $D(i)$ based on two factors: $d(i, 0)$ and $e(i, 0) . d(i, 0)$ has been computed in step 5 , and $e(i, 0)$ can be obtained locally. Then it calculates the added delay using formula (7) or (8).

Step 7: Check the current time $t$ : if $t<D(i)$, go to step 1; else let $d(i, t)=d\left(i, t_{j}\right)$.

Step 8: If $d(i, t) \leq n$, node $i$ abandons its attempt to rebroadcast; else rebroadcasts $M_{s}$ to all its neighbors.

Step 9: The algorithm ends.

\section{Performance Evaluation}

To verify the proposed $\mathrm{ML}^{2} \mathrm{~B}$, we made lots of simulations with an 802.11 MAC layer, using NS-2 [21]. Nodes in MANET are placed randomly in a 2-D square area. For all simulation results, CBR streams are used, and each broadcast stream consists of packets of size 512 bytes. In the all simulations made in this paper, we use the formula (7) to calculate the added delay. The abandoning threshold and energy threshold used in our simulations are configured as: $n=b / 5, E_{T}=E^{\prime} / 100 . b$ is the average number of neighbors of nodes.

\subsection{Performance Metrics Used in Simulations}

- Saved rebroadcast (SRB): $(x-y) / x$, where $x$ is the number of nodes that receive the broadcasted message, and $y$ is the number of nodes that rebroadcasts the message after their reception of the message. Therefore SRB of OBM is 0 under all scenarios.

- Reachability (RE): $x / z$, where $z$ is the number of all nodes in the simulated connected network. So RE is also known as the coverage rate. 
- Maximum end-to-end delay (MED): the interval form the time the broadcasted message is initiated to the time the last node in the network receiving the message.

- Life-time (LT): the interval from the time the network is initiated to the time the first node dies.

\subsection{Simulation Results}

\subsubsection{Performance Dependence on the Network Scale}

To study the influence of network scale on $\mathrm{ML}^{2} \mathrm{~B}$, we maintain a same node density by placing randomly different number of nodes separately in square areas of different size. The packets generation rate here is 2 packets-per-second (pps). As illustrated in Fig.2, $\mathrm{ML}^{2} \mathrm{~B}$ achieves high SRB without sacrificing the RE and MED under varying network sizes. According to expectation, MED increases with the increased network scale.

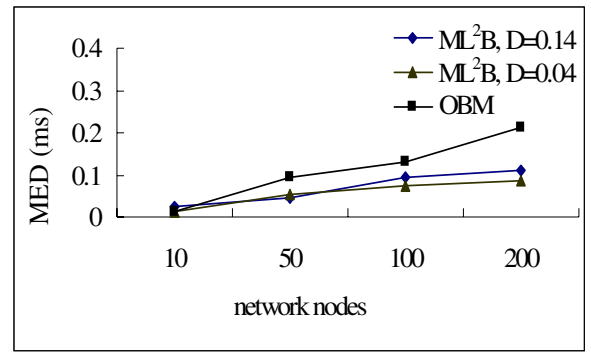

(a)

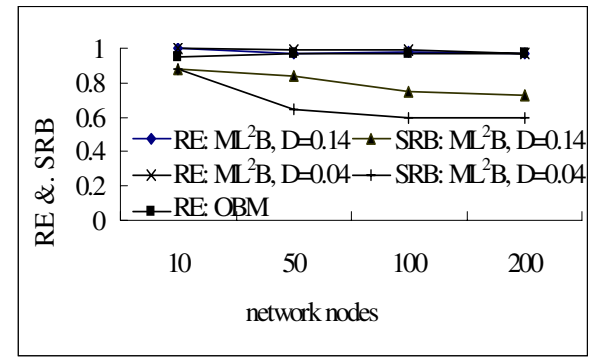

(b)

Fig. 2. Influence of network scale

\subsubsection{Performance Dependence on Node Density}

Many simulations were made to study the $\mathrm{ML}^{2} \mathrm{~B}$ performance. For the reason of limited pages, we give the results of the network consisting of 50 nodes, which is shown by Fig.3. The packets generation rate here is 2 pps. Results illustrated by Fig. 3 shows SRB of $\mathrm{ML}^{2} \mathrm{~B}$ falls with the decrease of node density. That is because the theoretical

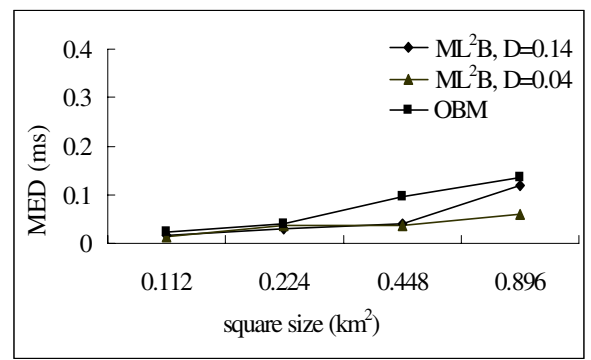

(a)

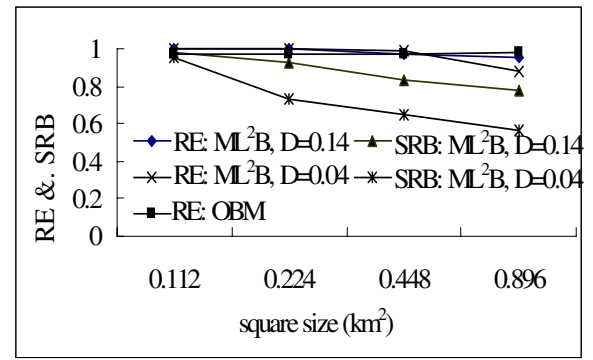

(b)

Fig. 3. Influence of node density 
value of SRB depends upon the node density. Large density causes big SRB, and ideal SRB will be zero when the node density is below a certain threshold, which is not the main issue of this paper.

We also compare the performance of $\mathrm{ML}^{2} \mathrm{~B}$ with maximum added delay $D=0.14 \mathrm{~s}$ and $D=0.04$ s. From Fig.2 and Fig.3, it is clear that the former outbalanced the latter in SRB and RE. And both of them have less MED than the OBM in all circumstances. Therefore, in the following experiments we set $D=0.14 \mathrm{~s}$.

\subsubsection{Performance Dependence on Packets Generation Rate}

We study the influence of network load on network performance by varying the packets generation rate from 2 pps to 10 pps. Simulation results in Fig. 4 show that increased network load incurs little impact on $\mathrm{ML}^{2} \mathrm{~B}$, however leads to increased MED in OBM. $\mathrm{ML}^{2} \mathrm{~B}$ maintains nearly as high RE as OBM, and simultaneously achieves SRB with a value larger than $80 \%$, which reveals the superiority of $\mathrm{ML}^{2} \mathrm{~B}$ over OBM.

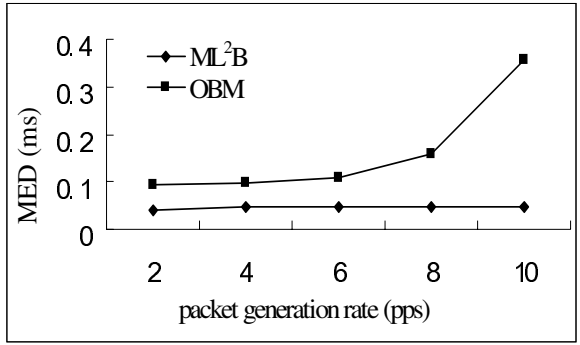

(a)

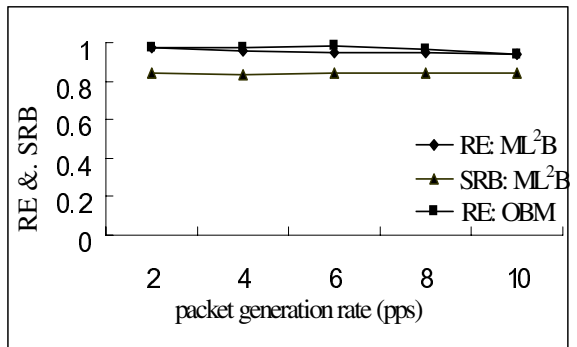

(b)

Fig. 4. Influence of network load

It can be summarized from the above simulations that, $\mathrm{ML}^{2} \mathrm{~B}$ achieves high $\mathrm{SRB}$ without sacrificing the RE and MED under all circumstances. It is beyond our expectation that $\mathrm{ML}^{2} \mathrm{~B}$, which has delayed the rebroadcast for an interval of $D(i)$, obtains a smaller MED than OBM that has not delayed rebroadcast. Using the different $D(i)$ values for different nodes, $\mathrm{ML}^{2} \mathrm{~B}$ greatly alleviates and avoids the contention and its resulting collision problem that persecutes OBM seriously, thus making $\mathrm{ML}^{2} \mathrm{~B}$ achieve a smaller maximum end-to-end delay than OBM. In a word, $\mathrm{ML}^{2} \mathrm{~B}$ could effectively relieve the broadcast storm problem.

\subsubsection{Life-Time Evaluation}

Fig. 5 shows the network life-time of $\mathrm{OBM}$ and $\mathrm{ML}^{2} \mathrm{~B}$ under the same scenario, in which each node's initial energy is uniformly distributed between $0.5 \mathrm{~J}$ (Joule) and 1.0 J. The first and last node dies separately at $32.48 \mathrm{~s}$ and $33.62 \mathrm{~s}$ in OBM. After $33.62 \mathrm{~s}$ no node dies due to malfunction of the broadcast caused by the unconnectivity of MANET due to too many dead nodes. While in $\mathrm{ML}^{2} \mathrm{~B}$, they happen at $73.05 \mathrm{~s}$ and $95.0 \mathrm{~s}$ separately. Life-time is defined as the interval from the time MANET is initiated to the 


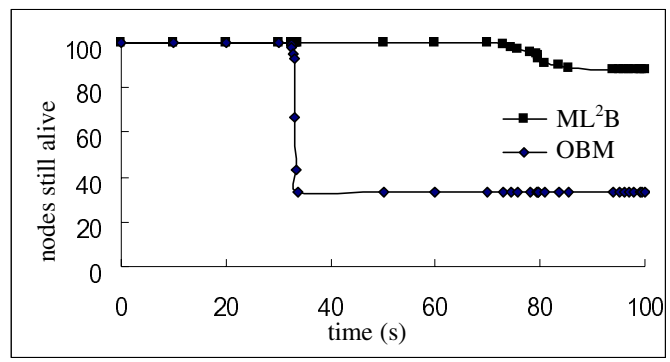

Fig. 5. Nodes still alive in the network of 100 nodes

time the first node dies. Obviously, $\mathrm{ML}^{2} \mathrm{~B}$ has more than doubles the useful network life-time compared with OBM.

We break the whole simulation time into many small time steps which also are called as rounds. Broadcast originator broadcasts each packet to other nodes in the network during each round. Table 1 shows the network life-time by rounds with different initial energy, which manifests $\mathrm{ML}^{2} \mathrm{~B}$ obtains much longer network life-time than OBM.

Table 1. Life-time using different amount of initial energy

\begin{tabular}{ccc}
\hline Initial Energy(J/node) & Protocol & Life-Time (rounds) \\
& ML $^{2} \mathrm{~B}$ & 192 \\
0.25 & OBM $^{2}$ & 45 \\
\multirow{2}{*}{0.5} & ML $^{2} \mathrm{~B}$ & 245 \\
& OBM & 91 \\
\multirow{2}{*}{1.0} & ML $^{2} \mathrm{~B}$ & 407 \\
& OBM & 195 \\
\hline
\end{tabular}

\section{Conclusion}

To solve the broadcast storm problem and maximize the network life-time, we propose an efficient broadcast protocol $\mathrm{ML}^{2} \mathrm{~B}$ for MANET. $\mathrm{ML}^{2} \mathrm{~B}$ is a novel added delay strategy, in which each node requires only its one-hop local information to fulfill a broadcast task. The thorough distributed and localized nature of $\mathrm{ML}^{2} \mathrm{~B}$ results in less overhead than ordinary broadcast methods. It is featured by the following properties: localized algorithm to cut down overhead, effective coverage adaptive to reduce broadcast redundancy and energy-efficient to maximize network lifetime. The effectiveness of $\mathrm{ML}^{2} \mathrm{~B}$ has been verified by simulations in NS-2.

\section{References}

1. Ni, S.Y., Tseng, Y.C., Chen, Y.S., Sheu, J.P.: The Broadcast Storm problem in a Mobile Ad Hoc Network. In: Proceedings of the 5th ACM/ IEEE Int. Conf. on Mobile Computing and Networking, pp. 151-162. IEEE, Seattle (1999) 
2. Pagani, E., Possi, G.P.: Providing Reliable and Fault Tolerant Broadcast Delivery in Mobile Ad-hoc Networks. Mobile Networks and Applications, 175-192 (1999)

3. Peng, W., Lu, X.C.: On the Reduction of Broadcast Redundancy in Mobile Ad Hoc Networks. In: Proceedings of MobiHoc, pp. 129-130 (2000)

4. Lin, C.R., Gerla, M.: Adaptive Clustering for Mobile Wireless Networks. IEEE JSAC, 1265-1275 (1997)

5. Sun, M.T., Lai, T.H.: Location Aided Broadcast in Wireless Ad Hoc Network Systems. In: Proceedings of IEEE WCNC, pp. 597-602. IEEE Computer Society Press, Los Alamitos (2002)

6. Wu, J., Lou, W.: Extended multipoint relays to determine connected dominating sets in MANETs. In: Proceedings of SECON, pp. 621-630 (2004)

7. Katsaros, D., Manolopoulos, Y.: The Geodesic Broadcast Scheme for Wireless Ad Hoc Networks. In: Proceedings of WoWMoM'06 (2006)

8. Wu, J., Dai, F.: A generic distributed broadcast scheme in ad hoc wireless networks. IEEE TC 53, 1343-1354 (2004)

9. Wieselthier, J.E., Nguyen, G.D., Ephremides, A.: Algorithm for Energy-Efficient Multicasting in Static Ad Hoc Wireless Networks. Mobile Networks and Applications 6, 251-263 (2001)

10. Wieselthier, J.E., Nguyen, G.D., Ephremides, A.: On the Construction of Energy-Efficient Broadcast and Multicast Trees in Wireless Networks. In: Proceedings of IEEE INFOCOM, IEEE Computer Society Press, Los Alamitos (2000)

11. Wan, P.J., Calinescu, G.X., Li, Y., Frieder, O.: Minimum-Energy Broadcast Routing in Static Ad Hoc Wireless Networks. In: Proceedings of IEEE INFOCOM (2001)

12. Cheng, M.X., Sun, J., Min, M., Du, D.Z.: Energy Efficient Broadcast and Multicast Routing in Ad Hoc Wireless Networks. In: Proceedings of 22nd IEEE Int'l Performance, Computing, and Comm. Conf. (2003)

13. Egecioglu, O., Gonzalez, T.F.: Minimum-Energy Broadcast in Simple Graphs with Limited Node Power. In: Proceedings of PDCS, pp. 334-338 (2001)

14. Cagalj, M., Hubaux, J.P., Enz, C.: Minimum-Energy Broadcast in All-Wireless Networks: NP-Completeness and Distribution Issues. In: Proceedings of MOBICOM (2002)

15. Liang, W.: Constructing Minimum-Energy Broadcast Trees in Wireless Ad Hoc Networks. In: Proceedings of MOBIHOC (2002)

16. Li, D., Jia, X., Liu, H.: Minimum Energy-Cost Broadcast Routing in static Ad Hoc Wireless Networks. IEEE Transactions on Mobile Computing 3(2) (2004)

17. Agarwal, M., Cho, J.H., Gao, L., Wu, J.: Energy Efficient Broadcast in Wireless Ad hoc Networks with Hitch-hiking. In: Proceedings of IEEE INFOCOM, IEEE Computer Society Press, Los Alamitos (2004)

18. Song, W.Z., Li, X.Y., Wang, W.Z.: Localized Topology Control for Unicast and Broadcast in Wireless Ad Hoc Networks. IEEE Transactions on Parallel and Distributed Systems 17, 321-334 (2006)

19. Ingelrest, F., Simplot-Ryl, D.: Localized Broadcast Incremental Power Protocol for Wireless Ad Hoc Networks. In: Proceedings of IEEE ISCC, IEEE Computer Society Press, Los Alamitos (2005)

20. Mauve, M., Widmer, J., Hartenstein, H.: A Survey on Position-Based Routing in Mobile Ad Hoc Networks. IEEE Network 30-39 (2001)

21. NS-2 Network Simulator (2006), http://isi.edu/nsnam/ns/index.html 